\title{
The Application of a Complex Composite Fractal Interpolation Algorithm in the Seabed Terrain Simulation
}

\author{
Chongyang Lv and Fei Yu $(\mathbb{D}$ \\ College of Automation, Harbin Engineering University, Harbin 150001, China \\ Correspondence should be addressed to Fei Yu; yufei414@126.com
}

Received 13 April 2018; Revised 18 June 2018; Accepted 21 June 2018; Published 9 July 2018

Academic Editor: Ivan Giorgio

Copyright (C) 2018 Chongyang Lv and Fei Yu. This is an open access article distributed under the Creative Commons Attribution License, which permits unrestricted use, distribution, and reproduction in any medium, provided the original work is properly cited.

\begin{abstract}
Seabed terrain modelling is one of the key technologies in the Subsea Environmental Information System, and this system is critical for underwater vehicle path planning. A composite fractal interpolation algorithm based on improved fractional Brownian motion (FBM) and an improved iterative function system (IFS) is proposed in this paper to increase the precision of the seabed terrain model for submarine topography and to account for the complexity and irregularity of fractal properties in each region. The MATLAB simulation experiment showed that fractal properties of the model built by the complex composite fractal interpolation algorithm were closer to real surface features. After calculation analysis, the model built by the complex composite fractal interpolation algorithm, when compared with the model built by the traditional interpolation algorithm or by the single fractal interpolation algorithm, had higher precision and was more suitable for path planning for underwater vehicles.
\end{abstract}

\section{Introduction}

Three-dimensional seabed terrain modelling is helpful to simulate the real seabed surface and to verify the static path planning technique for underwater vehicles under the sea [1]. It is very difficult to obtain the altitude data of the seabed, which requires substantial financial and material resources; thus, we use an interpolation method to establish a seabed terrain model based on the limited scattered data points [1].

D.G. Krige, a South African scientist, first proposed the interpolation algorithm [2]. The Kriging interpolation algorithm has higher precision when compared with the traditional linear interpolation algorithm. This algorithm is still a smooth interpolation method with high geometric accuracy [3]. A surface modelled by the Kriging interpolation algorithm is smooth, whereas the real surface is disorganized and rugged. The model constructed with the Kriging interpolation algorithm hides the most important features of the real surface $[4,5]$. Therefore, it is necessary to find an interpolation algorithm that can show real characteristics of the surface.

In the 1970s, Mandelbrot, a famous mathematician, proposed fractal theory. The real surface can be approximated using fractal geometry; therefore, fractal interpolation is an effective tool [6]. Although the fractal interpolation algorithm based on fractional Brownian motion (FBM) has a single algorithm parameter and strong randomness, presently, it is most commonly used [7, 8]. M. F. Barnsley, an American mathematician, proposed the fractal interpolation algorithm based on IFS. The terrain model established by this fractal interpolation algorithm solves the previous problem well [9]. However, the fractal interpolation algorithm based on IFS cannot directly process the discrete data; it can only build a terrain model based on regular grid data [9].

The original elevation data points of the seabed are scattered. This paper presents a composite fractal interpolation algorithm based on the improved FBM and IFS to establish a seabed terrain model that can reflect the real surface characteristics. The first step was to perform interpolation on the original elevation data of the seabed using the fractal interpolation algorithm of the improved FBM and then to obtain the preliminary seabed terrain model by improving the selection method of random quantity. The next step was to obtain regular grid data by interpolation processing to control the general direction and outline of the terrain. Finally, a local self-similar final seabed terrain model was established 


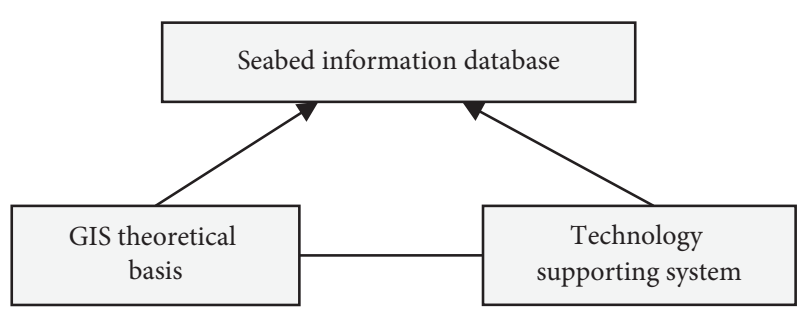

FIGURE 1: Core content diagram.

using regular grid data based on the improved IFS fractal interpolation.

\section{Subsea Environmental Information System}

The Subsea Environmental Information System is not only the application of GIS in the marine field but is also the result of the integration of science and earth science information [10]. This system contains various geological phenomena, geophysical field characteristics, and distribution of geographical features and attributes. This system is the embodiment of strategic thinking [11]. This system contains three core contents including the seabed information database, a GIS theoretical basis, and a technology supporting system [11] (Figure 1).

The structure of this system can be divided into three layers including the database layer, the application system layer, and the user terminal [12]. The database layer mainly refers to the standard seabed information database that is integrated. The application system layer is the centre of data scheduling, query, statistics, and access, as well as the establishment of the system model based on the seabed information database. The user terminal contains three levels including maintenance layer users, professional layer users, and browsing layer users [12].

This paper focuses on the establishment of the system model in the application system layer. The marine environment is complex and dynamic, and the seabed terrain undulates. The difficulty of measuring ocean data has increased dramatically, and the available baseline data is limited and scattered [13]. Due to the above factors, it is very difficult to establish an underwater terrain model with greater certainty and to be able to show real seabed terrain features. Satellite imagery of the data area was used in this paper (Figure 2).

Meanwhile, the contour map of this area, obtained from the 2009 Chinese electronic chart, can be seen in Figure 3.

\section{Simulation Method and Process}

3.1. Fractal Interpolation and Improvement Based on Fractional Brownian Motion (FBM). FBM can be generated in many ways, such as the midpoint displacement; this is the simplest and most classic method [14]. The important point of random midpoint displacement method is the determination of the random displacement quantity. In terrain modelling, the size of this random displacement is related to the statistical characteristics of the elevation data, such as how

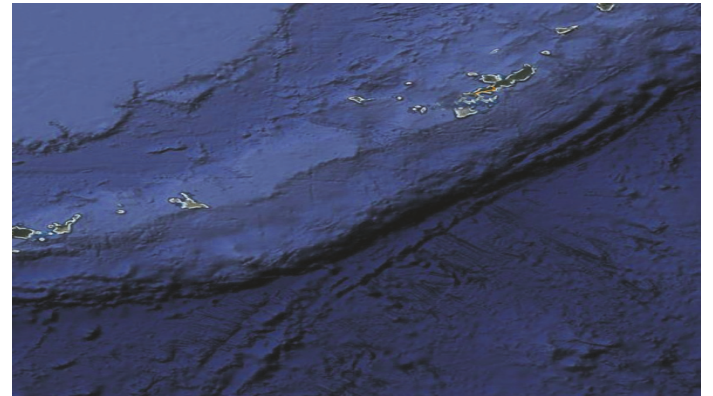

FIGURE 2: Satellite imagery of the study area.

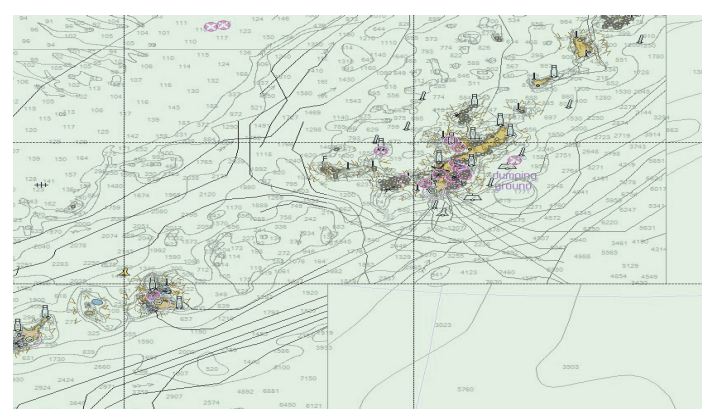

Figure 3: Contour map.

to describe the self-similar parameter of rough terrain or the mean and standard deviation of the elevation data. For terrain changes, the terrain model flattened out from rough when the index increased. The least squares method was used to calculate the magnitude of the random displacement. The calculation formula is as follows:

$$
\Delta i=d_{i}^{H} \times \delta \times \sqrt{1-2^{2 H-2}} \times \operatorname{gauss}()
$$

where $i$ is the iterative times of random midpoint displacement and $d_{i}$ is the distance between two points after the $i$ iteration.

Although the rhombus was the basic unit interpolated, there were "crease problems" in all these measures. "Crease problems" represent a constant common edge between two adjacent units, and the midpoint of this common edge has two different elevation values. A cross-section finally appears on the terrain model [15]. The simplest way to solve this problem is to use a line segment to take the midpoint elevation value. The key point is the connection and selection of the line segments.

The original data contained many scattered points, and the best way to manage these data was to connect them using a triangular irregular network (TIN) that can describe terrain surfaces at different levels of resolution [16]. Compared with the grid model, the TIN model can show a complex terrain surface more effectively. Previous research indicates that the Delaunay triangular network is the most prominent in terrain fitting among all TIN [16]. The performance of random midpoint displacement interpolation based on the Delaunay triangular network can achieve better results. The improved random midpoint displacement method not only maintains 


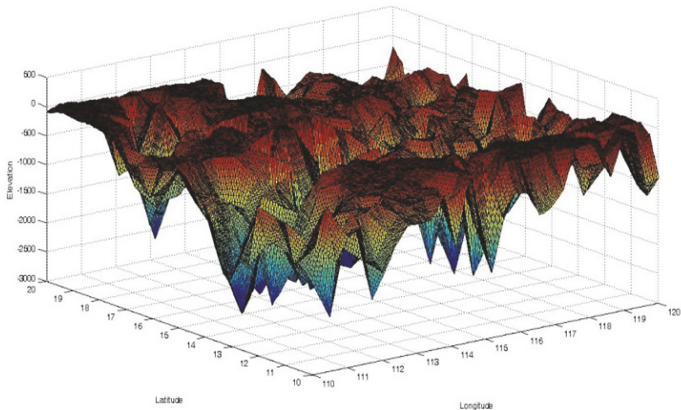

FIGURE 4: Three iterations of the terrain model.

the advantages of the Delaunay triangular network for fitting topography but also solves the "crease problem."

The selection of the scale-free zones and the $H$ index is relatively unitary. This paper intends to use statistical analysis of the terrain data to determine the range of the scalefree zone. The entire data interval was divided into several communities based on the scale-free zone; then, the $H$ index and $\delta$ parameter were determined separately.

The method to select the minizone is shown below. For $\mathrm{A}(\mathrm{x}, \mathrm{y})$, near the centre of the entire interval plane, the elevation value is $\mathrm{Z}$. The influence of point $\left(x_{i}, y_{i}, z_{i}\right)$ on point A is related to d, the plane distance between two points. $\sigma$ is the relevance between two points. When the distance $\mathrm{d}$ increased, $\sigma$ exponentially decreased. The relevance $\sigma=1$ when two points are close together and when the elevation of these two points is the same.

$$
\sigma=e^{-d^{2}}
$$

The relationship between relevance $\sigma$ and distance $\mathrm{d}$ is reflected well in this function. For a given threshold $\omega$, we assumed that there is no interaction between two points when the relevance $\sigma<\omega$.

Among all the points $\left(x_{i}, y_{i}\right)$ that related to point $(x, y)$, the farthest point was defined as $d_{\max }$, the midpoint as $A(x, y)$, and the length of the side as $2 d_{\max }$. A rectangle was drawn to find index $H$ and parameter $\delta$ of the data point in this area, and the random displacement of this region was calculated.

The sea area coverage of the original data points in this paper was the north latitude $10.11^{\circ}-29.6^{\circ}$ and the east longitude $120.11^{\circ}-132.8^{\circ}$. The interpolation terrain model in this region was constructed by the random midpoint displacement method, which improved with three iterations (Figure 4).

The random displacement measured by this method is better than the single random displacement of the whole region. It takes too long to run three or more iterations due to the large quantity of data, the time complexity, and the space complexity. Meanwhile, insufficient memory in the computing platform results in a large error. Too few iterations, however, will result in low resolution of the terrain model and will enlarge the random amplification in this model.

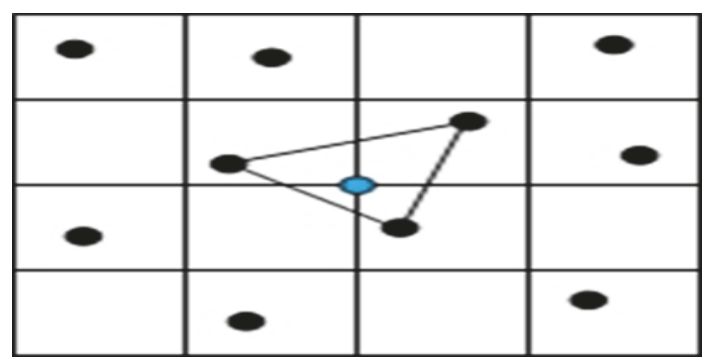

FIGURE 5: Grid interpolation.

Therefore, it is necessary to conduct more detailed modelling based on this model.

3.2. Acquisition of Regular Grid Data. The fractal interpolation based on IFS is an important method for further modelling, but it can only handle regular grid data. The processing of data is necessary.

The seabed elevation data is obtained after iteration calculation from the preliminary model. These data are interpolated to get the required regular grid data. Triangle interpolation was used in this research and the algorithm steps were as follows:

(1) The scattered data points were found in the grid after dividing the whole data area grid and the grid interval was $t$.

(2) The grid node is defined as the centre and the Matts, whose side is $2 t$, as the unit; then, a search was applied for the data point $\mathrm{n}$ in the unit.

(3) If $n \geq 3$, three points were taken to build a triangle that contains the centre node in Matts and the triangle with the smallest area was chosen; then, the elevation value of the plane made by the interpolation node in this triangle was calculated. The result was the interpolation elevation value of the node.

(4) If $n<3$, a grid interval was added to the scope of the search unit, the search for data points continued until $\mathrm{n} \geq 3$, and the interpolation elevation value of the node was calculated.

The interpolation process for the triangle is shown in Figure 5.

The blue grid node was within the triangle formed by the three data points. Another interval was added if the data points within Matts were less than 3, until three data points within the triangle were found.

Compared with the triangular mesh interpolation algorithm, the search of the grid nodes and the maker in the triangle mesh were omitted in this interpolation algorithm. By finding the triangle through grid nodes, the algorithm was simplified and the programming was easier to implement. Rule grid data was obtained based on the preliminary terrain model through the interpolation algorithm. The range of rule grid data was 22-28 degrees north latitude and 124-130 degrees east longitude. The topographic map by grid data is presented in Figure 6. 


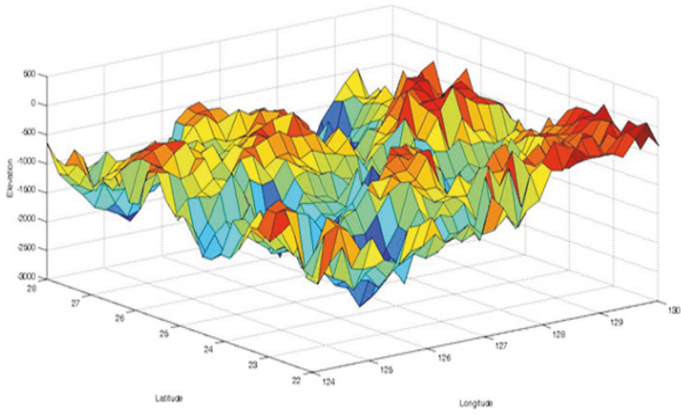

FIGURE 6: Regular grid diagram.

3.3. Fractal Interpolation and Improvement Based on the Iterative Function System (IFS). The IFS fractal interpolation is based on the self-similarity principle of fractal [17].

Regular grid data was previously obtained, and $I$ is the data point set. Compressional transformation was adopted on $x, y$, and $z$ directions, $\left(x_{n}, y_{m}, z_{n, m}\right)$ and is defined as the point set: $I ; n=0,1, \cdots, N ; m=0,1, \cdots, M$ and $a \leq x_{n} \leq b, c \leq$ $y_{m} \leq d . \Delta x$ and $\Delta y$ are step-size and divided into grids, as follows:

$$
\begin{aligned}
& a=x_{0}<x_{1}<\cdots<x_{N}=b \\
& c=y_{0}<y_{1}<\cdots<y_{M}=d
\end{aligned}
$$

After calculation and derivation, the compression transformation of each direction is as follows:

$$
\begin{gathered}
\phi_{n}(x)=x_{n-1}+\frac{\left(x_{n}-x_{n-1}\right)\left(x-x_{0}\right)}{x_{N}-x_{0}} \\
\varphi_{m}(y)=y_{m-1}+\frac{\left(y_{m}-y_{m-1}\right)\left(y-y_{0}\right)}{y_{M}-y_{0}} \\
F_{n, m}(x, y, z)=e_{n, m} x+f_{n, m} y+g_{n, m} x y+\alpha_{n, m} z+k_{n, m}
\end{gathered}
$$

And

$$
\begin{aligned}
g_{n, m}= & \frac{z_{n-1, m-1}-z_{n-1, m}-z_{n, m-1}+z_{n, m}}{x_{0} y_{0}-x_{N} y_{0}-x_{0} y_{M}+x_{N} y_{M}} \\
& -\frac{\alpha_{n, m}\left(z_{0,0}-z_{N, 0}-z_{0, M}+z_{N, M}\right)}{x_{0} y_{0}-x_{N} y_{0}-x_{0} y_{M}+x_{N} y_{M}} \\
e_{n, m}= & \frac{z_{n-1, m-1}-z_{n, m-1}-\alpha_{n, m}\left(z_{0,0}-z_{N, 0}\right)}{x_{0}-x_{N}} \\
& -\frac{g_{n, m}\left(x_{0} y_{0}-x_{N} y_{0}\right)}{x_{0}-x_{N}} \\
f_{n, m}= & \frac{z_{n-1, m-1}-z_{n-1, m}-\alpha_{n, m}\left(z_{0,0}-z_{0, M}\right)}{y_{0}-y_{M}} \\
& -\frac{g_{n, m}\left(x_{0} y_{0}-x_{0} y_{M}\right)}{y_{0}-y_{M}}
\end{aligned}
$$

$$
\begin{aligned}
k_{n, m}= & z_{n, m}-e_{n, m} x_{N}-f_{n, m} y_{M}-\alpha_{n, m} z_{N, M} \\
& -g_{n, m} x_{N} y_{M}
\end{aligned}
$$

The compression transformation of $x$ and $y$ was determined. The vertical scale factor was defined by adding a free variable $\alpha$ to the compression transformation of $z(0 \leq \alpha<1)$. The role of the vertical scale factor was to control the surface roughness of the terrain. The more $\alpha$ grows, the rougher the terrain is and the more serrated the surface is [17].

Iterative operation was used on these three compression transformations. Based on the contracting mapping principle, we can get a fixed point known as the attractor of the IFS, and the image of this attractor represents the threedimensional seabed topographic map.

When the overall data area is larger, the changes in seabed topography are larger (gentle or rugged). It is not reasonable to use the $\alpha$ value to reflect the whole seabed topography. The model is closer to the real terrain if the whole area is divided into small pieces and then the $\alpha$ value for each small piece is calculated [17].

The vertical scale factor $\alpha$ represents the compression ratio for that feature of the terrain change, mapped from the whole area to a smaller range. The coefficient of variation was used to calculate the vertical scaling factor $\omega$ for the local plots in this research. The algorithm of $\omega$ is shown below.

As for $\left(x_{i}, y_{i}, z_{i}\right)$, the grid data on a local interval, the least square method was used to fit the linear equation of trend surface.

$$
\widehat{z}=b_{0}+b_{1} x+b_{2} y
$$

where $\widehat{z}_{i}\left(x_{i}, y_{i}\right)$ was the trend elevation value for each point in the region,

which was used to define

$$
\overline{\widehat{z}}=\frac{\sum_{i=1}^{n} \widehat{z}_{i}\left(x_{i}, y_{i}\right)}{n}
$$

where $\overline{\widehat{z}}$ is recorded as the overall average trend in elevation.

As is known, $z_{i}\left(x_{i}, y_{i}\right)$ is the real elevation value of each grid node and

$$
e_{i}=z_{i}-\widehat{z}_{i}
$$

which was used to define

$$
\delta=\sqrt{\frac{\sum_{i=1}^{n} e_{i}^{2}}{n}}
$$

Take $\delta$ as the similar standard deviation of the value of grid node elevation in the region.

The vertical scale factor $\omega$ was considered as

$$
\omega=\frac{\delta}{\overline{\widehat{z}}}
$$

Compared with the selection method that uses deviation alone, the selection method of vertical scale factor relates to 


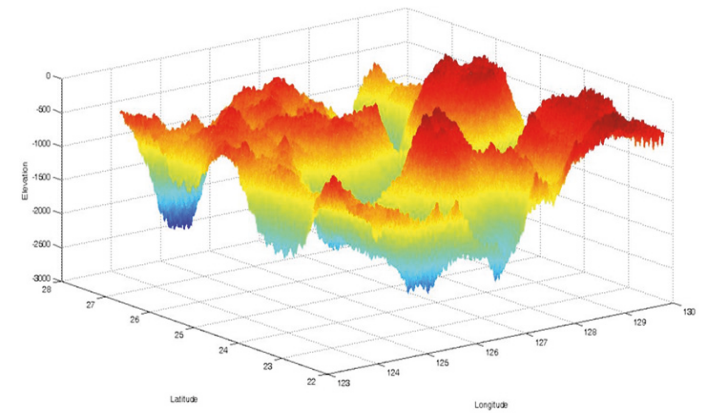

FIGURE 7: Terrain model of composite fractal interpolation.

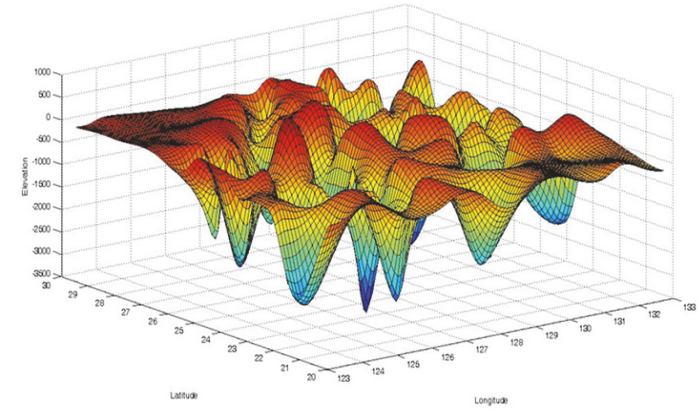

FIGURE 8: Traditional linear interpolation model.

the similar standard deviation and mean values for data point elevation values in the region. It not only accounts for characteristics of the grid data but also the geomorphic change trend in the region. The selection method of the vertical scale factor can reflect the characteristics of topography changes in the region.

\section{Results and Discussion}

4.1. Simulation Results. As previously shown, after preliminary modelling, the original scattered seabed altitude data in this area was derived from rule grid data; finally, the required altitude data of rule grid was obtained. The final seabed terrain model was obtained from an improved IFS interpolation algorithm (Figure 7).

The seabed terrain model was established by traditional linear interpolation and satellite images of this area, as shown in Figure 8.

When compared with the traditional linear interpolation model or FBM fractal interpolation model, the composite fractal interpolation model, with higher resolution, had more realistic terrain features.

4.2. Results Analysis. Contour reconstruction method was used in this paper for the quantitative analysis comparison between the composite fractal interpolation model and the traditional linear interpolation model. Bathymetric charts of the composite fractal interpolation model and the traditional

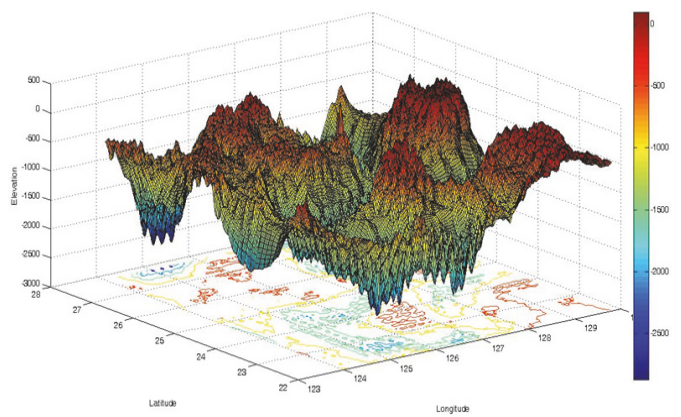

FIGURE 9: Bathymetric chart of the composite fractal interpolation model.

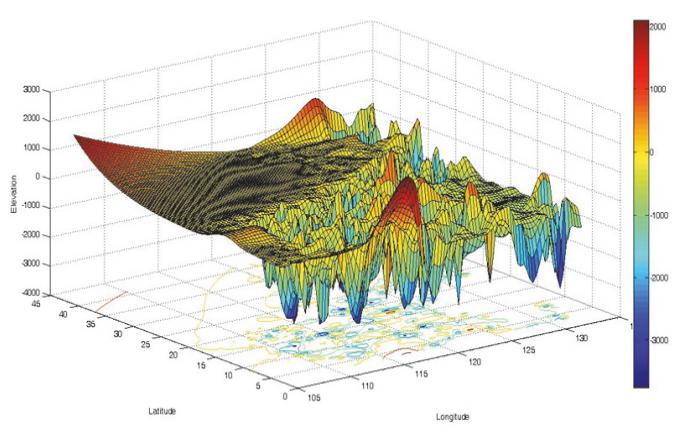

FIGURE 10: Bathymetric chart of the traditional linear interpolation model.

linear interpolation model are presented in Figures 9 and 10, respectively.

The contour map of this region obtained from the 2009 Chinese electronic chart represents the "truth value" contour line. The error value was calculated by comparing the contour lines for the first two interpolation models at contour lines of $-500 \mathrm{~m},-1000 \mathrm{~m}$, and $-1500 \mathrm{~m}$. The maximum error, mean error, and standard deviation of each group were then calculated (Figure 11).

Figure 11 shows that the maximum error for the two models was not significantly different. Whereas the average error for the composite fractal interpolation model was better than that of the traditional linear interpolation model, the standard deviation for the composite fractal interpolation model was larger than that of the traditional linear interpolation model at $-500 \mathrm{~m}$ and $-1000 \mathrm{~m}$. The main reason for this situation may be that the topography of this region undulates. Partial fractal dimension is used in the composite fractal interpolation model. The topographics are very close to the real surface of the earth, but they resulted in a large error for terrain altitude.

\section{Conclusions}

We conclude that good visualization effects and better precision were obtained from the seabed terrain model built by the composite fractal interpolation algorithm. Compared 


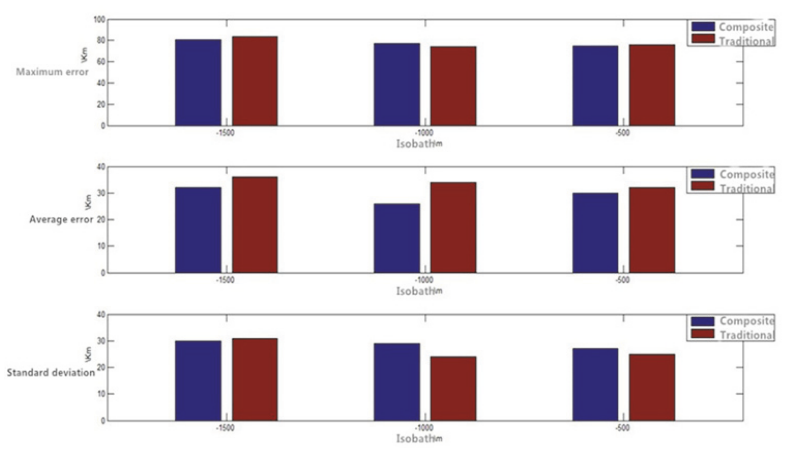

FIGURE 11: Error analysis.

with the traditional linear interpolation algorithm, topographic features of the model built by the composite fractal interpolation algorithm were much closer to the real surface of the earth. This model better reflects the complexity and irregularity of the seabed features and is more suitable for planning paths for underwater vehicles.

\section{Data Availability}

The data used to support the findings of this study have not been made available because these true data relate to the security of homeland geographic information and should be kept confidential.

\section{Conflicts of Interest}

The authors declare that there are no conflicts of interest regarding the publication of this paper.

\section{Acknowledgments}

This work was supported by the National Natural Science Foundation of China. (no. 51679047).

\section{References}

[1] W. U. Wen, Y. X. Zhou, L. I. Xing et al., "3D underwater terrain simulation using fractional Brownian motion," Computer Engineering \& Applications, vol. 46, no. 25, pp. 242-245, 2006.

[2] P. Chen, Y. Li, Y. Su, and X. Chen, "The improved kriging interpolation algorithm for local underwater terrain based on fractal compensation," Mathematical Problems in Engineering, vol. 2014, Article ID 289521, 8 pages, 2014.

[3] C.-T. Lai, S.-S. Hsiao, H.-M. Fang, and E. H. Wang, "The kriging cloud computing framework: Interpolation of topography by cloud computing with the kriging algorithm," Journal of Marine Science and Technology (Taiwan), vol. 23, no. 4, pp. 534-540, 2015.

[4] X. F. Zhi, X. Ma, G. L. Chen, and G. H. Li, "A modeling method of $3 \mathrm{D}$ visualization of submarine pipeline's virtual terrain based on ArcGIS and Unity3D," Energy Education Science and Technology Part A: Energy Science and Research, vol. 32, no. 4, pp. 2783-2796, 2014.

[5] P. Jiang, F. Liu, J. Wang, and Y. Song, "Cuckoo search-designated fractal interpolation functions with winner combination for estimating missing values in time series," Applied Mathematical Modelling: Simulation and Computation for Engineering and Environmental Systems, vol. 40, no. 23-24, pp. 9692-9718, 2016.

[6] P. Paramanathan and R. Uthayakumar, "Fractal interpolation on the Koch curve," Computers and Mathematics with Applications, vol. 59, no. 10, pp. 3229-3233, 2010.

[7] Q. Z. Li and X. R. Gao, "Generation and visualization of 3-D realistic natural terrain based on FBM," in Proceedings of the 4th International Conference on Image and Graphics, ICIG 2007, pp. 915-919, August 2007.

[8] L. Liu, X. Wang, and H. Ren, "3D seabed terrain establishment based on moving fractal interpolation," in Proceedings of the 7th International Joint Conference on Computational Sciences and Optimization, pp. 6-10, IEEE, July 2014.

[9] S. Wang and F. Kang, "Automatic optimization for large-scale real-time coastal water simulation," Mathematical Problems in Engineering, vol. 2016, Article ID 9034649, 9 pages, 2016.

[10] M. Greene, "Opening opportunities in subsea communications," in Proceedings of the OCEANS 2016, pp. 1-5, 2016.

[11] W. Z. Shi, B. Wang, and Y. Tian, "Accuracy analysis of digital elevation model relating to spatial resolution and terrain slope by bilinear interpolation," Mathematical Geosciences, vol. 46, no. 4, pp. 445-481, 2014.

[12] M. Toy, "Cloud services architectures," Procedia Computer Science, vol. 61, pp. 213-220, 2015.

[13] E. Tarameshloo, M. H. Loorak, P. W. L. Fong, and S. Carpendale, "Using visualization to explore original and anonymized LBSN data," Computer Graphics Forum, vol. 35, no. 3, pp. 291-300, 2016.

[14] M. F. Barnsley, "Fractal functions and interpolation," Constructive Approximation, vol. 2, no. 1, pp. 303-329, 1986.

[15] R. Ramstedt and J. Smed, "Midpoint displacement in multifractal terrain generation," in Proceedings of the International Conference on Intelligent Games and Simulation, 2016.

[16] B. Wessel, M. Huber, C. Wohlfart et al., "Accuracy assessment of the global TanDEM-X digital elevation model with GPS data," Isprs Journal of Photogrammetry \& Remote Sensing, vol. 139, pp. 171-182, 2018.

[17] C. M. Wittenbrink, "IFS fractal interpolation for 2D and 3D visualization," in Proceedings of the 6th Conference on Visualization '95, pp. 77-84, 1995. 


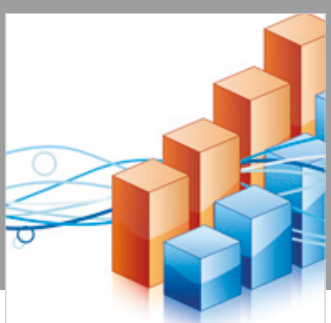

Advances in

Operations Research

\section{-n-m}
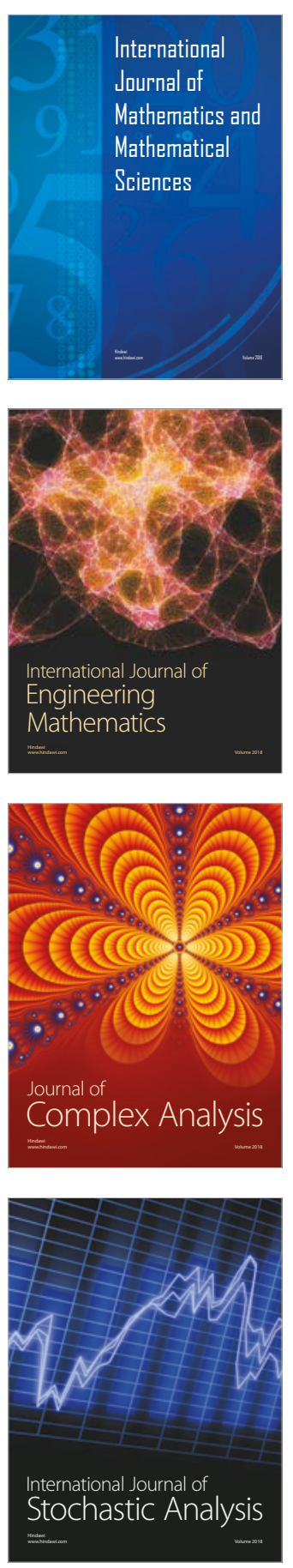
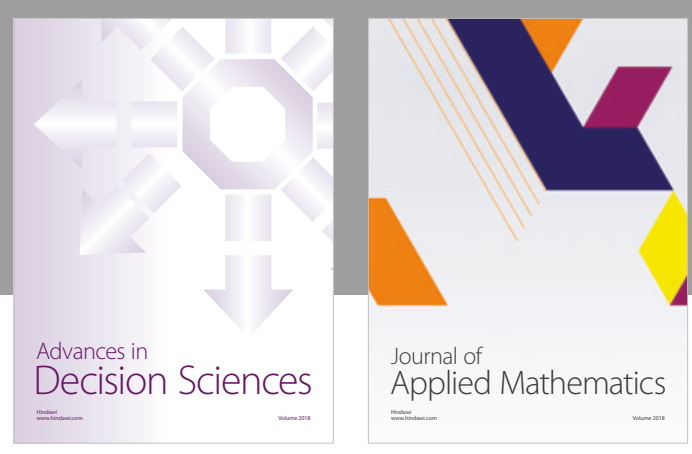

Journal of

Applied Mathematics
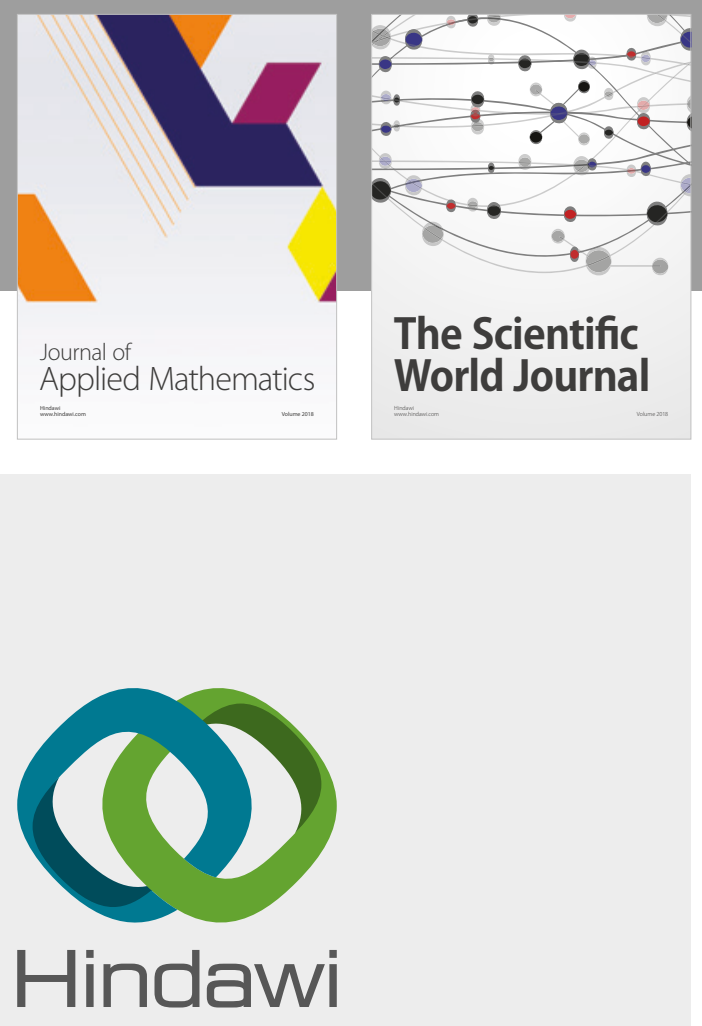

Submit your manuscripts at

www.hindawi.com

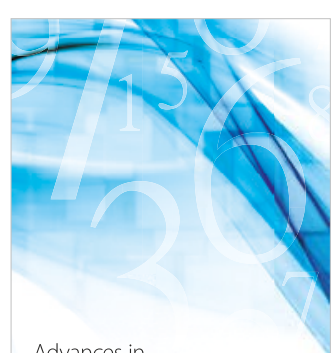

Advances in
Numerical Analysis
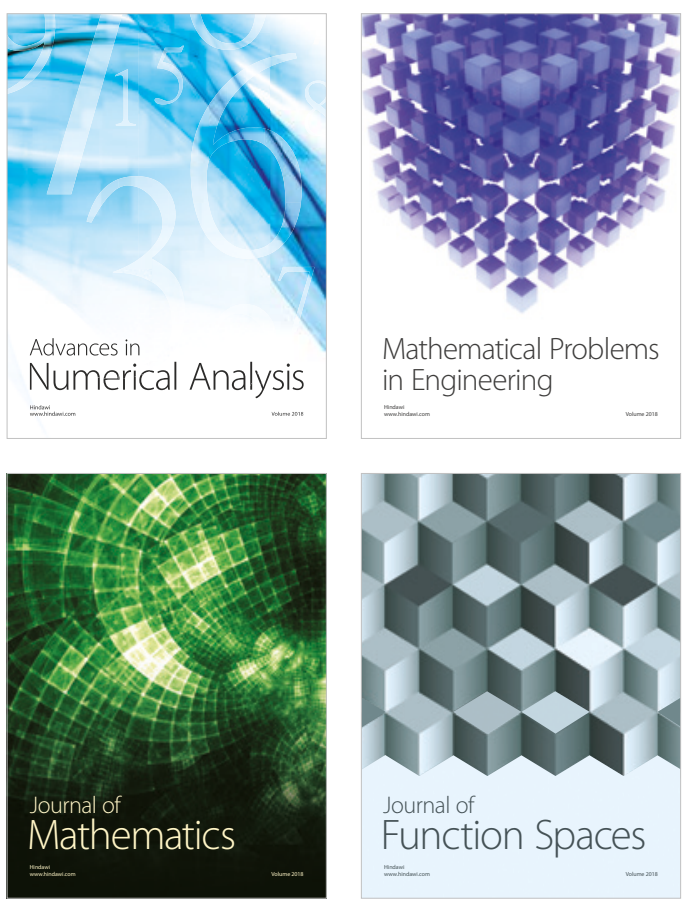

Mathematical Problems in Engineering

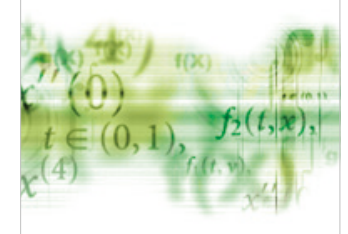

International Journal of

Differential Equations

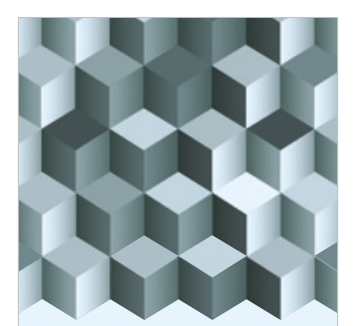

Journal of

Function Spaces

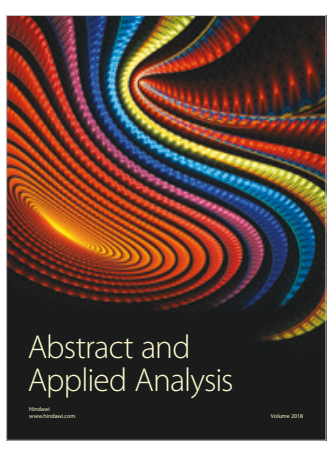

The Scientific

World Journal

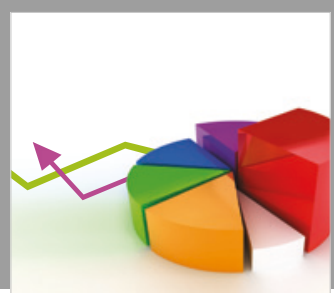

Journal of

Probability and Statistics
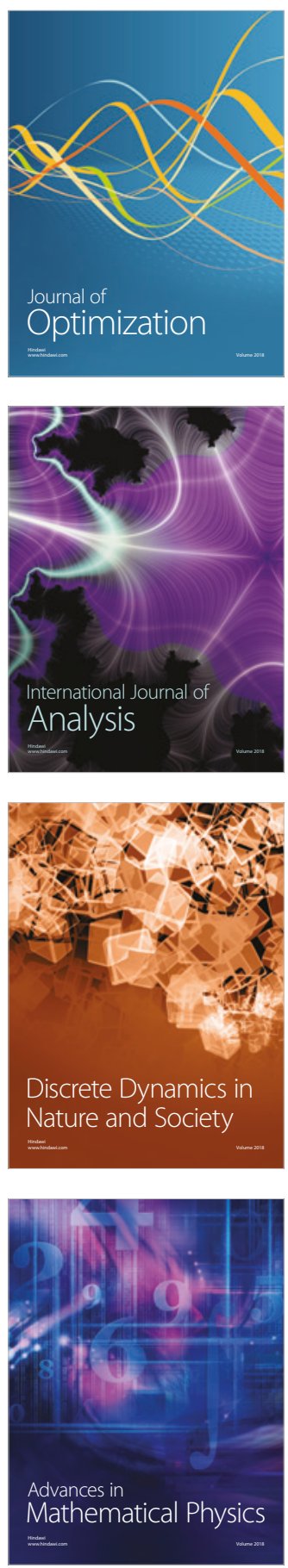